\title{
miR-124 promotes the neuronal differentiation of mouse inner ear neural stem cells
}

\author{
DI JIANG ${ }^{1,2^{*}}$, JINTAO DU ${ }^{1,3^{*}}$, XUEMEI ZHANG ${ }^{1,4}$, WEI ZHOU ${ }^{1}$, LIN ZONG $^{1,5}$, CHANG DONG $^{1}$,

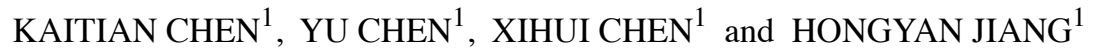

\begin{abstract}
${ }^{1}$ Department of Otolaryngology, The First Affiliated Hospital, and Institute of Otorhinolaryngology, Sun Yat-sen University, Guangzhou, Guangdong 510080; ${ }^{2}$ Department of Otolaryngology, People's Hospital of Dongguan, Dongguan, Guangdong 510080; ${ }^{3}$ Department of Otolaryngology, West China Hospital of Sichuan University, Chengdu, Sichuan 6100041; ${ }^{4}$ Department of Otorhinolaryngology, The Second Hospital of Hebei Medical University, Shijiazhuang, Hebei 050000; ${ }^{5}$ Department of Otorhinolaryngology, The Second Affiliated Hospital, Guangzhou Medical University, Guangzhou, Guangdong 510260, P.R. China
\end{abstract}

Received October 11, 2015; Accepted August 8, 2016

DOI: $10.3892 / \mathrm{ijmm} .2016 .2751$

\begin{abstract}
MicroRNAs (miRNAs or miRs) act as key regulators in neuronal development, synaptic morphogenesis and plasticity. However, their role in the neuronal differentiation of inner ear neural stem cells (NSCs) remains unclear. In this study, 6 miRNAs were selected and their expression patterns during the neuronal differentiation of inner ear NSCs were examined by RT-qPCR. We demonstrated that the culture of spiral ganglion stem cells present in the inner ears of newborn mice gave rise to neurons in vitro. The expression patterns of miR-124, miR-132, miR-134, miR-20a, miR-17-5p and miR-30a-5p were examined during a 14-day neuronal differentiation period. We found that miR-124 promoted the neuronal differentiation of and neurite outgrowth in mouse inner ear NSCs, and that the changes in the expression of tropomyosin receptor kinase $\mathrm{B}$ (TrkB) and cell division control protein 42 homolog (Cdc42) during inner ear NSC differentiation were associated with miR-124 expression. Our findings indicate that miR-124 plays a role in the neuronal differentiation of inner ear NSCs. This finding may lead to the development of novel strategies for restoring hearing in neurodegenerative diseases.
\end{abstract}

Correspondence to: Dr Hongyan Jiang or Dr Xihui Chen, Department of Otolaryngology, The First Affiliated Hospital of Sun-Yat Sen University, 58 Zhongshan Second Road, Guangzhou, Guangdong 510080, P.R. China

E-mail: hongy@mail.sysu.edu.cn

E-mail: earchen@126.com

${ }^{*}$ Contributed equally

Key words: inner ear, neural stem cells, microRNA-124, neuronal differentiation, hearing

\section{Introduction}

Sensorineural hearing loss is a severe hearing impairment that affects millions of individuals worldwide. Moreover, neuronal regeneration is uncommon in the mature inner ear $(1,2)$, in contrast to neurogenesis in the adult brain, such as in the subventricular zone (SVZ) of the lateral ventricles and the dentate gyrus of the hippocampus (3). Inner ear neural stem cells (NSCs) may exist in the spiral ganglion. Since NSCs can proliferate and differentiate into neurons (4-6), they may be used to regenerate damaged neurons in the inner ear. However, the intrinsic 'self-repair' capacity of inner ear NSCs is inactive in vivo for unclear reasons (unsuitable microenvironment, lack of guidance, etc.). Therefore, successfully harnessing the differentiation potential of inner ear NSCs may restore hearing. While the molecular mechanisms underlying the neuronal differentiation of inner ear NSCs are not yet fully understood, microRNAs (miRNAs or miRs) may play an important role in this process.

miRNAs are small non-coding RNAs that affect mRNA stability or inhibit translation by binding complementary sequences in the 3'-translated regions (3'-UTRs) of target mRNAs (7-10). Thus, miRNAs regulate multiple biological functions, including cell proliferation, differentiation and apoptosis $(11,12)$. miRNA expression studies in mammals using microarrays and reverse transcription-quantitative PCR (RT-qPCR) have demonstrated that miRNAs are expressed in the developing nervous system and in mature neurons (13-18). miRNAs have crucial functions in neuronal development and plasticity $(19,20)$. The miR-17 family has been shown to play an integral role in the regulation of neuronal differentiation (21). miR-124 expression has been shown to be upregulated during neuronal differentiation $(22,23)$. Several lines of evidence have indicated that miR-124 regulates neuronal differentiation by inhibiting small C-terminal domain phosphatase (SCP1), a component of the RE1-silencing transcription factor (REST)/ neuron-restrictive silencer factor (NRSF) transcriptional repression complex, and by targeting polypyrimidine tract 
binding protein 1 (PTBP1), a global repressor of brain-specific alternative splicing $(24,25)$. Moreover, global miRNA expression profiling by microarray analysis, RT-qPCR, and/or in situ hybridization have revealed several miRNAs with distinct spatio-temporal expression patterns in the embryonic and post-natal mouse inner ear (25-28). While miRNAs regulate the development, morphogenesis and function of the inner ear (29-33), it is not yet known whether miRNAs also regulate the neuronal differentiation of inner ear NSCs.

In this study, 6 miRNAs (miR-124, miR-132, miR-134, miR-20a, miR-17-5p and miR-30a-5p) were selected based on their involvement in neuronal differentiation/neurogenesis as discussed in the 'Introduction' and in the 'Discussion'; their expression patterns during the neuronal differentiation of inner ear NSCs were examined by RT-qPCR. Our data demonstrate that miR-124 is important for the differentiation of inner ear NSCs into neurons. Our results revealed that miR-124 expression is upregulated during neuronal differentiation. Moreover, miR-124 increased the percentage of cells expressing neuronspecific class III $\beta$-tubulin in culture, and increased the neurite length in mouse inner ear NSCs. These changes were accompanied by changes in the expression of tropomyosin receptor kinase $\mathrm{B}(\mathrm{TrkB})$ and cell division control protein 42 homolog $(\mathrm{Cdc} 42)$. TrkB is a receptor of brain-derived neurotrophic factor (BDNF), and participates in the regulation of neurogenesis, neurite outgrowth and in the survival of spiral ganglion neurons. The small GTP-ase, Cdc42, which regulates both microtubules and actin filaments in a number of cells, regulates the neurite extension of spiral ganglion neurons. Our data demonstrated that miR-124 promotes the neuronal differentiation of and neurite outgrowth in inner ear NSCs, and regulates the expression of TrkB and Cdc42 in inner ear NSCs.

\section{Materials and methods}

Animals. Post-natal day 1 (P1) C57BL/6 mice ( $\mathrm{n}=135$; Laboratory Animal Center of Sun Yat-sen University, Guangzhou, China) were used for the experiments. The animals were sacrificed according to policies set forth by the Animal Care and Use Committee of Sun Yat-sen University based on the National Institutes of Health guidelines for animal care. All animal experiments were also approved the Animal Care and Use Committee of Sun Yat-sen University.

Inner ear NSC cultures, neuronal differentiation and immunostaining. The isolation and culture of NSCs from the spiral ganglia of newborn C57BL/6 mice were performed as previously described (4). Spiral ganglia, isolated from 5 mice, were digested in $200 \mu \mathrm{l}$ of $0.125 \%$ trypsin in phosphate-buffered saline (PBS; Gibco/Thermo Fisher Scientific, Waltham, MA, USA) at $37^{\circ} \mathrm{C}$ for $15 \mathrm{~min}$. The cells were carefully triturated with plastic $200 \mu \mathrm{l}$ pipette tips, centrifuged and suspended in $2 \mathrm{ml}$ Dulbecco's modified Eagle's medium (DMEM)/high glucose and F12 medium (mixed 1:1; Gibco/Thermo Fisher Scientific) supplemented with N2 and B27 supplements (Invitrogen/ Thermo Fisher Scientific), epidermal growth factor (EGF; $20 \mathrm{ng} / \mathrm{ml}$ ), basic fibroblast growth factor (bFGF; $20 \mathrm{ng} / \mathrm{ml}$ ), insulin-like growth factor 1 (IGF-1; $20 \mathrm{ng} / \mathrm{ml}$ ) (PeproTech, Rocky Hill, NJ, USA). The cell suspension was passed through a 70-mm cell strainer (BD Falcon, Franklin Lakes, NJ, USA) to remove cell clumps. For neuronal differentiation, mouse inner ear NSCs at 5 days were harvested and cultured in laminin and poly-L-lysine-coated dishes in DMEM-F12 (1:1) medium supplemented with $20 \mathrm{ng} / \mathrm{ml}$ of neurotrophin-3 (NT-3), $20 \mathrm{ng} / \mathrm{ml}$ of BDNF) (both from PeproTech), $10 \mathrm{ng} / \mathrm{ml}$ of leukemia inhibitory factor (LIF) (R\&D Systems, Inc., Minneapolis, MN, USA), $2 \mathrm{mM}$ of L-glutamine (Gibco/Thermo Fisher Scientific) and $3 \mathrm{mM}$ of $\mathrm{KCl}$ (Sigma, St. Louis, MO, USA) for 7 days. Half of the differentiation medium was replaced every other day. The NSCs were allowed to differentiate for up to 14 days (day 14). Cell proliferative ability was assessed by BrdU incorporation assay as previously described (34). Undifferentiated and differentiated inner ear NSCs were fixed with $4 \%$ paraformaldehyde and immunostained with antibodies specific for BrdU (C2181; 1:10,000; Sigma), Tuj1 (class III $\beta$-tubulin; MO15013; 1:250; Neuromics, Edina, MN, USA), p2 $7^{\text {Kip1 }}$ (554069; 1:150; BD Biosciences, San Jose, CA, USA), ATP-binding cassette sub-family G member 2 (Abcg2; sc-25822; 1:150; Santa Cruz Biotechnology, Inc., Dallas, TX, USA), Sox2 (SAB2104660;1:250; Sigma), Nestin (MO15012; 1:200; Neuromics), TrkB (ab18987; 1:200; Abcam, Cambridge, UK) and Cdc42 (sc-8401; 1:150 Santa Cruz Biotechnology, Inc.). Nuclei were counterstained with Fluoroshield Mounting Medium with 4',6-diamidino-2-phenylindole (DAPI; ab104139; Abcam).

$R N A$ isolation and reverse transcription-quantitative $P C R(R T-q P C R)$. The cells were collected in cold D-Hank's solution (Gibco/Thermo Fisher Scientific), and RNA was extracted using TRIzol reagent (Invitrogen/Thermo Fisher Scientific) and column-purified with an RNeasy kit [Qiagen (Suzhou) Translational Medicine Co., Ltd., Suzhou Industrial Park, China]. Reverse transcription was performed using M-MLV reverse transcriptase (Roche Diagnostics, Mannheim, Germany) according to the instructions provided by the manufacturer. The sequences of the primers were as follows: Nestin forward, 5'-GCCGAGCTGGAGCGCGA GTTAGAG-3' and reverse, 5'-GCAAGGGGGAAGAGAA GGATGTCG-3'; Sox2 forward, 5'-ACCCGGGCCTCAA CGCTCACG-3' and reverse, 5'-TCCCCTTCTCCAGTT CGCAGTCC-3'; p27 Kip1 forward, 5'-CTGGAGCGGAT GGACGCCAGAC-3' and reverse, 5'-CGTCTGCTCCAC AGTGCCAGC-3'; Abcg2 forward, 5'-GCTGTGGAGC TGTTCGTAGTGG-3' and reverse, 5'-GCTAAAGTA CTGAAGCCAGGAC-3'; Nanog forward, 5'-CCCTTCCC TCGCCATCACA-3' and reverse, 5'-ACCGCTTGC ACTTCATCCTTTG-3'; Tuj1 forward, 5'-TAGACCCCA GCGGCAACTAT-3' and reverse, 5'-GTTCCAGG TTCCAAGTCCACC-3'; and glyceraldehyde 3-phosphate dehydrogenase (GAPDH) forward, 5'-AACGGGAAG CCCATCACC-3' and reverse, 5'-CAGCCTTGGCAG CACCAG-3'. Primers were synthesized by Invitrogen. Transcripts of GAPDH were amplified and used as an internal reference. Quantitative PCR (qPCR) was performed using $2 \mu \mathrm{l}$ of SYBR-Green/ROX qPCR Master Mix (Suzhou GenePharma Co., Ltd., Suzhou, China). All procedures were subjected to a TaqMan miRNA assay (Applied Biosystem 7500) according to the manufacturer's instructoins. U6 was employed as an endogenous control (for miRNAs) to normalize data using the $2^{-\Delta \Delta \mathrm{Ct}}$ method. Probe and primers specific for 
miR-124, miR-132, miR-134, miR-20a, miR-17-5p, miR-30a-5p, or for U6 RNA internal control were purchased from LC Sciences (Hangzhou, China). PCR and qPCR analyses of at least 3 independent cultures were performed.

Western blot analysis. For protein extraction, the cells were washed twice and resuspended in lysis buffer containing $50 \mathrm{mM}$ of Tris- $\mathrm{HCl}, \mathrm{pH} 7.4,150 \mathrm{mM}$ of $\mathrm{NaCl}, 1 \% \mathrm{NP}-40$, $10 \%$ glycerol, $1 \mathrm{mM}$ of sodium orthovanadate, $1 \mathrm{mM}$ of PMSF and a protease inhibitor cocktail (Shanghai Roche Pharmaceutical Co. Ltd., Shanghai, China). Subsequently, $20 \mu \mathrm{g}$ of cell lysates were separated by $8-15 \%$ of resolving sodium dodecyl sulfate-polyacrylamide gel electrophoresis (SDS-PAGE), transferred onto polyvinylidene fluoride membranes, and immunoblotted with antibodies according to the manufacturer's instructions. The antibodies used in western blot analysis included $\beta$-actin (sc-47778; 1:3,000; Santa Cruz Biotechnology, Inc.), Tuj1 (MO15013; 1:1,000, Neuromics), TrkB (ab18987; 1:1,000; Abcam) and Cdc42 (sc-8401; 1:1,000; Santa Cruz Biotechnology, Inc.).

Electroporation. RNA oligoribonucleotides for miR-124 mimics (which mimic endogenous mature miR-124 molecules), non-specific miRNA (negative control) and miR-124 inhibitor [small interfering RNA (siRNA) targeting miR-124 (miR-124 siRNA)] were all purchased from Genepharma (Shanghai, China). For electroporation, mouse inner ear NSCs were dissociated into a single-cell suspension with $0.25 \%$ trypsin (Gibco/Thermo Fisher Scientific) at $37^{\circ} \mathrm{C}$ for $3 \mathrm{~min}$. Subsequently, $1 \times 10^{6}$ cells were electroporated with $5 \mathrm{~g}$ of miR-124 mimics, non-specific miRNA or miR-124 siRNA using an Amaxa Mouse NSC Nucleofector kit (Lonza Group Ltd., Basel, Switzerland) following the manufacturer's instructions. RNA oligoribonucleotides specific for enhanced green fluorescent protein (eGFP) were used to monitor electroporation efficiency. Electroporated cells were plated on cover glasses coated with poly-L-lysine and laminin (R\&D Systems, Inc.), and incubated in differentiation medium at $37^{\circ} \mathrm{C}$. The transfected inner ear NSCs were then subjected to neuronal differentiation for up to 3 days.

Measurement of neuron percentage and neurite length. Transfected inner ear NSCs were fixed and immunostained at 3 days post-differentiation. The neuron percentage was determined as the number of cells stained positive for the neuron-specific marker, Tuj1, divided by the number of cells stained with Hoechst 33342 (the total number of differentiated inner ear NSCs) from 15 randomly selected fields at x20 magnification. All neurons within the image frame were counted. Cells located in clumps were excluded. Images were acquired using an Olympus microscope [Olympus IX71 Inverted Microscope and Olympus FV1000 confocal microscope; Olympus Inc. (China), Guangzhou, China] and quantitative analysis was carried out using ImageJ software (each image of x20 magnification generally captures at least 200 differentiated inner ear NSCs).

As previously described (35), we measured the entire length of the longest neurite extending from 95 differentiated neurons (identified by the presence of Tuj1), which was selected randomly (using a 40x objective lens). Neurites that were not entirely in the frame were excluded. Neurite lengths were determined using Image J software. Processes that could not be distinguished from others were eliminated.

Statistical analysis. One-way ANOVA and Chi-square tests were used to analyze the data, and the differences between variables were considered to be statistically significance when the value was $\mathrm{P}<0.05$. All data are expressed as the means \pm SEM, unless otherwise stated.

\section{Results}

Culture and differentiation of inner ear NSCs. The cells were isolated from the mouse spiral ganglion and were cultured for 5 days. The cells formed floating solid neurospheres (Fig. 1A), which were positive for BrdU, Sox 2 and other stem cell markers, such as Nestin, p27 Kip1 and Abcg2 (Fig. 1B), confirming the self-renewal and proliferative capacity of these cells. To further characterize the stemness properties of the inner ear neurospheres, we confirmed the expression of markers of progenitor cells (Nestin, p27 ${ }^{\mathrm{Kip} 1}$, Abcg2 and Nanog) by RT-qPCR (Fig. 1C).

To initiate the differentiation of mouse inner ear NSCs into neurons, we plated the neurospheres onto a coated substratum in differentiation medium. Four hours after adherence, differentiated neurons that expressed neuron-specific class III $\beta$-tubulin (detected by Tuj1 antibody) began to sprout neurites from the attached neurospheres. Immunofluorescence staining demonstrated that the number of Tuj1-positive cells increased on day 3, and reached maximal levels on day 14 (Fig. 2A-D). To confirm the differentiation of NSCs, the expression of neuronspecific class III $\beta$-tubulin was examined by western blot analysis and by RT-qPCR (Fig. 2E-F). Proliferating spheres expressed low levels of neuron-specific class III $\beta$-tubulin, which is a marker of mature neurons. This phenomenon may be related to the differentiation of individual peripheral NSCs. During differentiation, the levels of neuron-specific class III $\beta$-tubulin increased on day 3 and reached the highest levels on day 14 , which was consistent with the data obtained by immunofluorescence.

TrkB and Cdc42 are upregulated during the neuronal differentiation of inner ear NSCs. Although Cdc42 and TrkB have been shown to affect neuronal differentiation and polarity (36-40), the role of TrkB and Cdc42 in the neuronal differentiation of mouse NSCs remains unknown. In this study, we cultured inner ear NSCs in differentiation medium for 14 days; our results revealed that $\mathrm{TrkB}$ and $\mathrm{Cdc} 42$ expression were low in the undifferentiated inner ear NSCs, and their expression gradually increased during differentiation (Fig. 3C-D). Since TrkB and Cdc42 modulate neuronal differentiation and neurite extension, TrkB and Cdc42 may be involved in the differentiation of inner ear NSCs into neurons.

Expression of miRNAs during the differentiation of inner ear NSCs. To determine the function of miRNAs in the neuronal differentiation of inner ear NSCs, we compared the expression levels of 6 miRNAs between undifferentiated and differentiated inner ear NSCs. A total of 6 miRNAs were selected based on their established role in neuronal differentiation. 


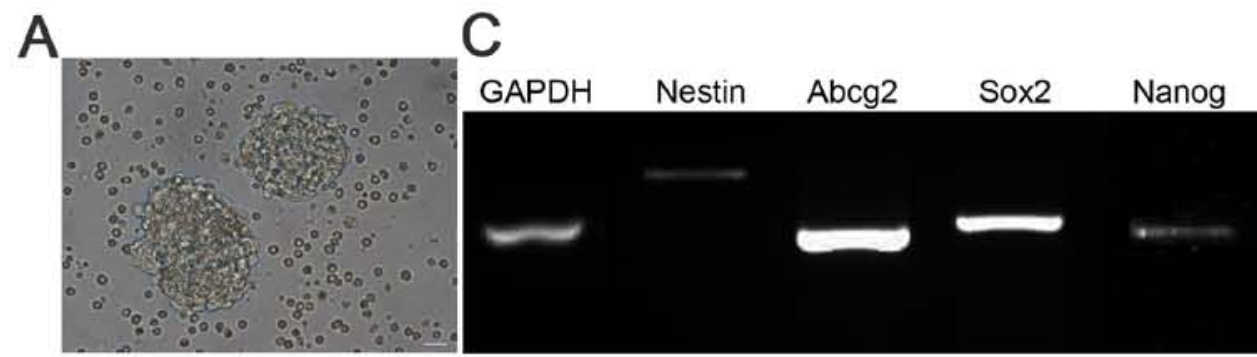

B

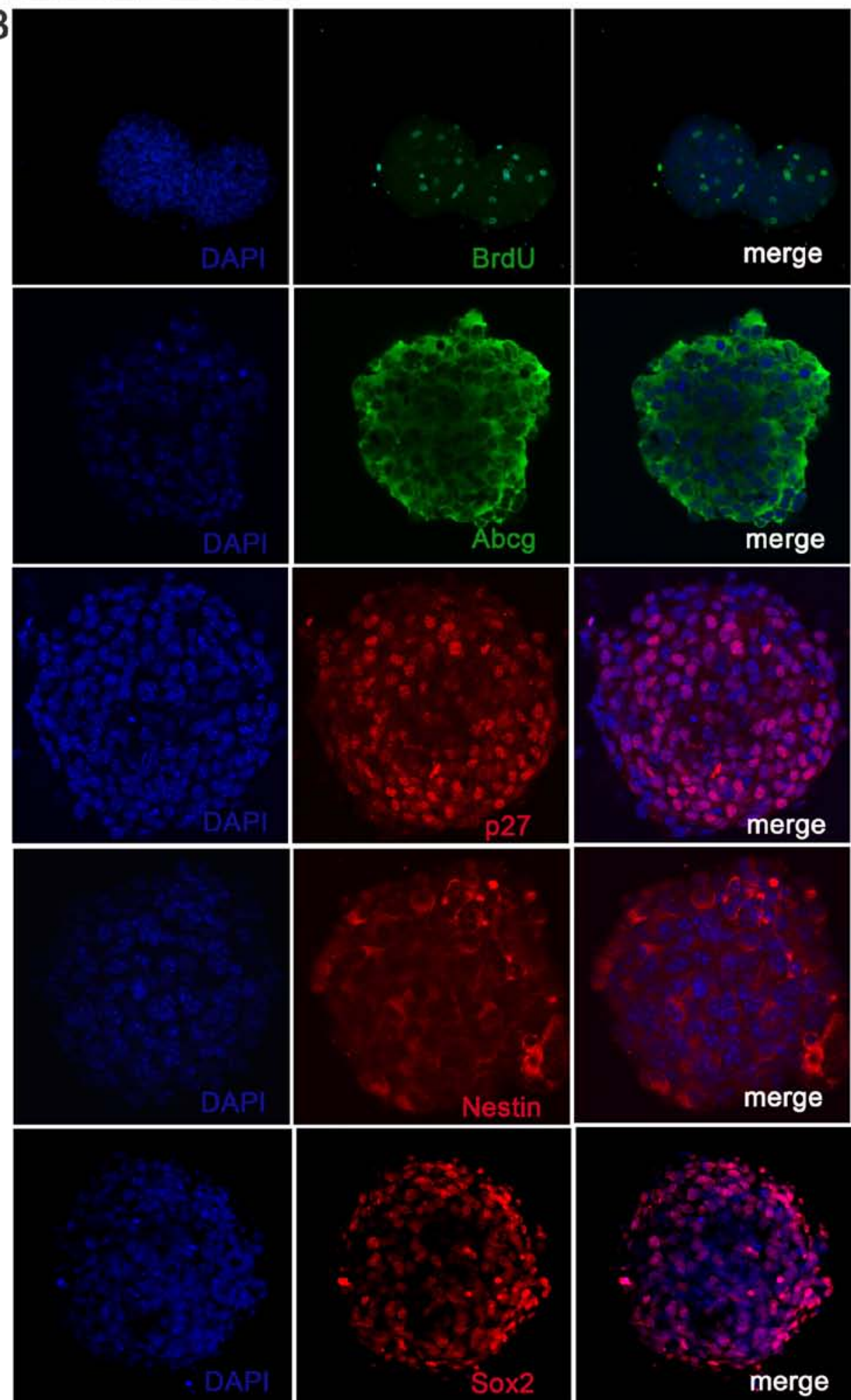

Figure 1. Inner ear neural stem cells (NSCs) isolated from newborn mice proliferate and form floating neurospheres. Neurospheres were immunostained after 5 days under proliferative conditions. (A) Typical morphology of a spiral ganglion-derived neurosphere after 5 days of culture in suspension. (B) Neurospheres were positive for BrdU, Sox2, Nestin, Abcg2 and p27 ${ }^{\text {Kipl }}$. (C) Analysis of stemness-related genes in inner ear NSCs by RT-qPCR. 

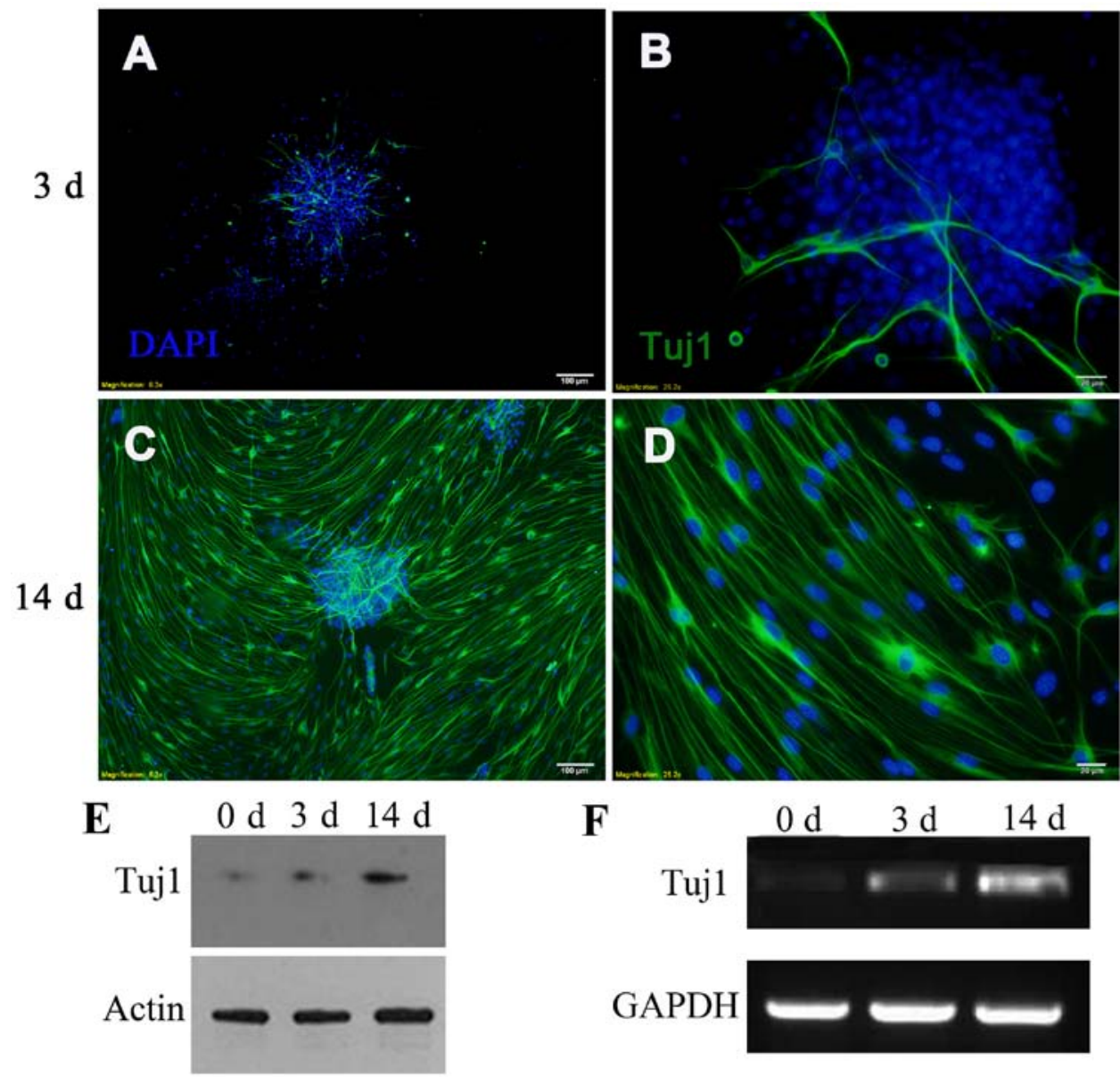

F

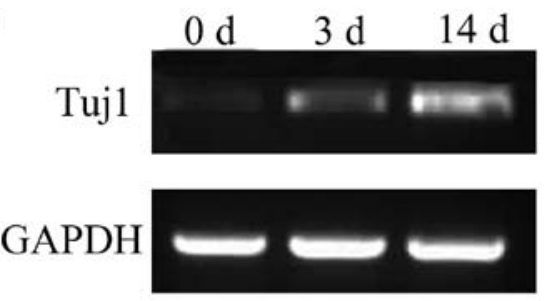

Figure 2. Tuj1 expression increases during neuronal stem cell (NSC) differentiation. (A) Two representative images of inner ear NSC differentiation (days 3 and 14). Mature neurons were visualized with Tuj1 (green), and the nuclei were visualized with 4',6-diamidino-2-phenylindole (DAPI) (blue). (A and C) Lower magnification (x10) scale bars, $100 \mu \mathrm{m}$; (B and D) higher magnification (x40) scale bars, $20 \mu \mathrm{m}$. Neuron-specific class III $\beta$-tubulin expressions (E) and mRNA that encode neuron-specific class III $\beta$-tubulin (F) in undifferentiated cells ( 0 days) and during differentiation ( 3 and 14 days). d, days.
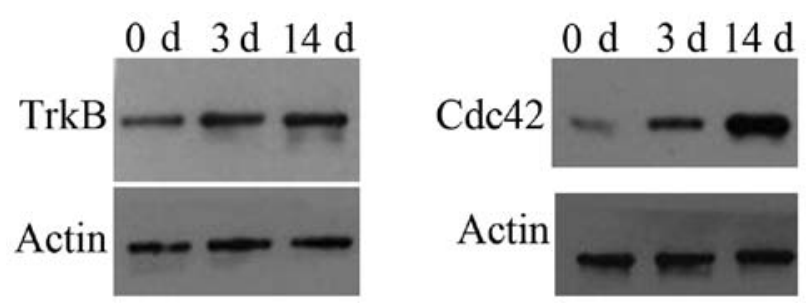

Figure 3. The expression of cell division control protein 42 homolog $(\mathrm{Cdc} 42)$ and tropomyosin receptor kinase B (TrkB) during the differentiation of inner ear neural stem cells (NSCs). Western blot analysis of Cdc42 and TrkB in undifferentiated ( 0 day) and differentiated NSCs ( 3 and 14 days). d, days.

We set the expression level in undifferentiated inner ear NSCs as the baseline, and compared it to the expression in differentiated inner ear NSCs on days 3 and 14 using the $2^{-\Delta \Delta \mathrm{Ct}}$ method. The levels of miR-17-5p decreased by day $3(\mathrm{p}<0.05)$ and increased by day $14(\mathrm{p}<0.05)$; however, the overall expression level of miR-17-5p did not show any significant change over the 14-day period (Fig. 4). Similarly, no significant change in miR-20a expression levels were observed during neuronal differentiation ( $p>0.05$ ). By contrast, the miR-30a-5p expression levels increased and the miR-134 levels significantly decreased during the differentiation of NSCs on day 14, suggesting that miR-30a-5p and miR-134 play a role in neuronal differentiation. The expression levels of 2 miRNAs, miR-124 and miR-132 were altered on days 3 and 14 of differentiation. The levels of miR-124 increased 6-fold, while the levels of miR-132 decreased 4-fold during the entire 14-day period. In this study, we focused on the role of miR-124 in the differentiation of inner ear NSCs.

miR-124 promotes the neuronal differentiation of and neurite outgrowth in inner ear NSCs. Inner ear NSCs were transfected with RNA oligoribonucleotides for miR-124 mimics, non-specific miRNA and miR-124 inhibitor. The transfection efficiency was 50-60\%, as determined by eGFP (Fig. 5A and B). In addition, we measured the miR-124 expression levels in differentiated inner ear NSCs by RT-qPCR. Three days following electroporation with miR-124 inhibitor, the miR-124 levels were decreased by 2.13-fold. By contrast, the electroporation of miR-124 mimics led to a 3.02-fold increase in miR-124 expression (Fig. 5C).

Next, we determined the effect of miR-124 on the percentage of neuron and neurite outgrowth. We found that the overexpression of miR-124 in differentiated inner ear NSCs increased neuronal differentiation and neurite outgrowth, whereas the knockdown of miR-124 had an opposite effect (Fig. 6A-C). The results revealed that at 3 days of differentia- 

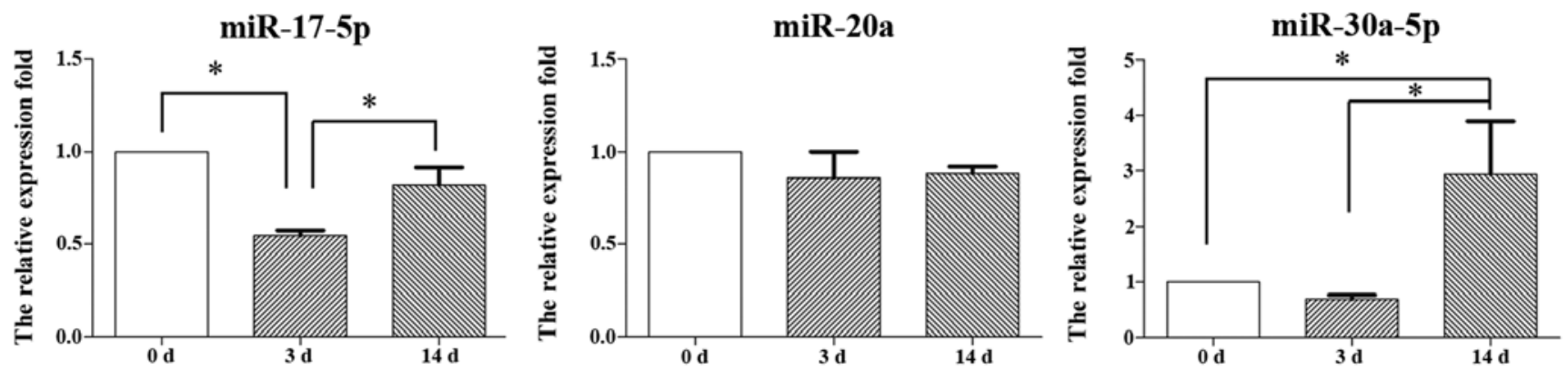

miR-134
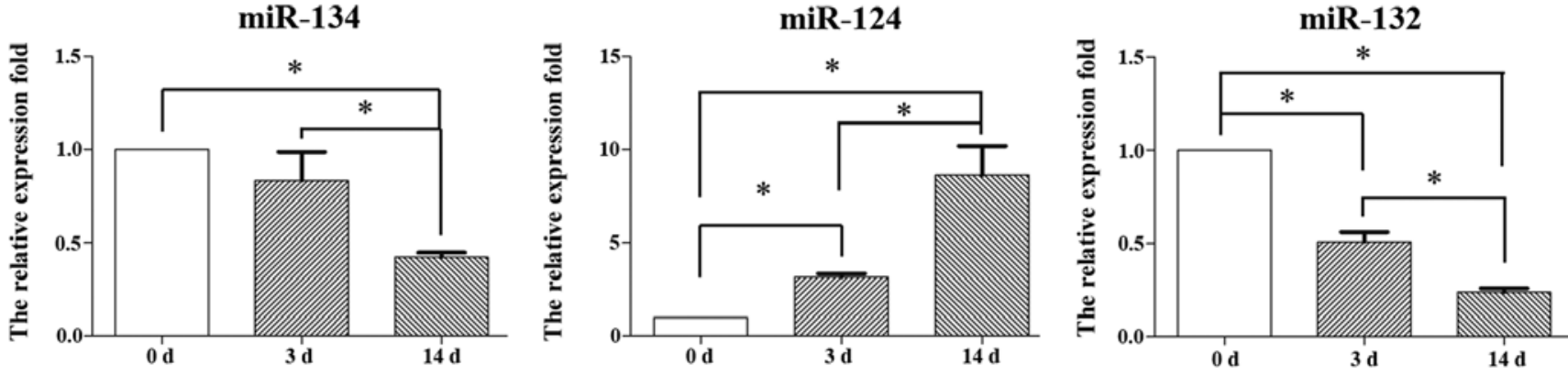

Figure 4. Results of RT-qPCR of the miRNA expression in mouse inner ear during the differentiation of neural stem cells (NSCs). Expression of miRNAs in undifferentiated (0 days) or differentiated ( 3 or 14 days) inner ear NSCs. The RT-qPCR data were normalized to U6 expression levels. The fold change was expressed as the ratio of miRNA expression in differentiated and undifferentiated cells (day 0 ) using the $2^{-\Delta \Delta C t}$ method. " $p<0.05$, statistically significant difference. d, days.
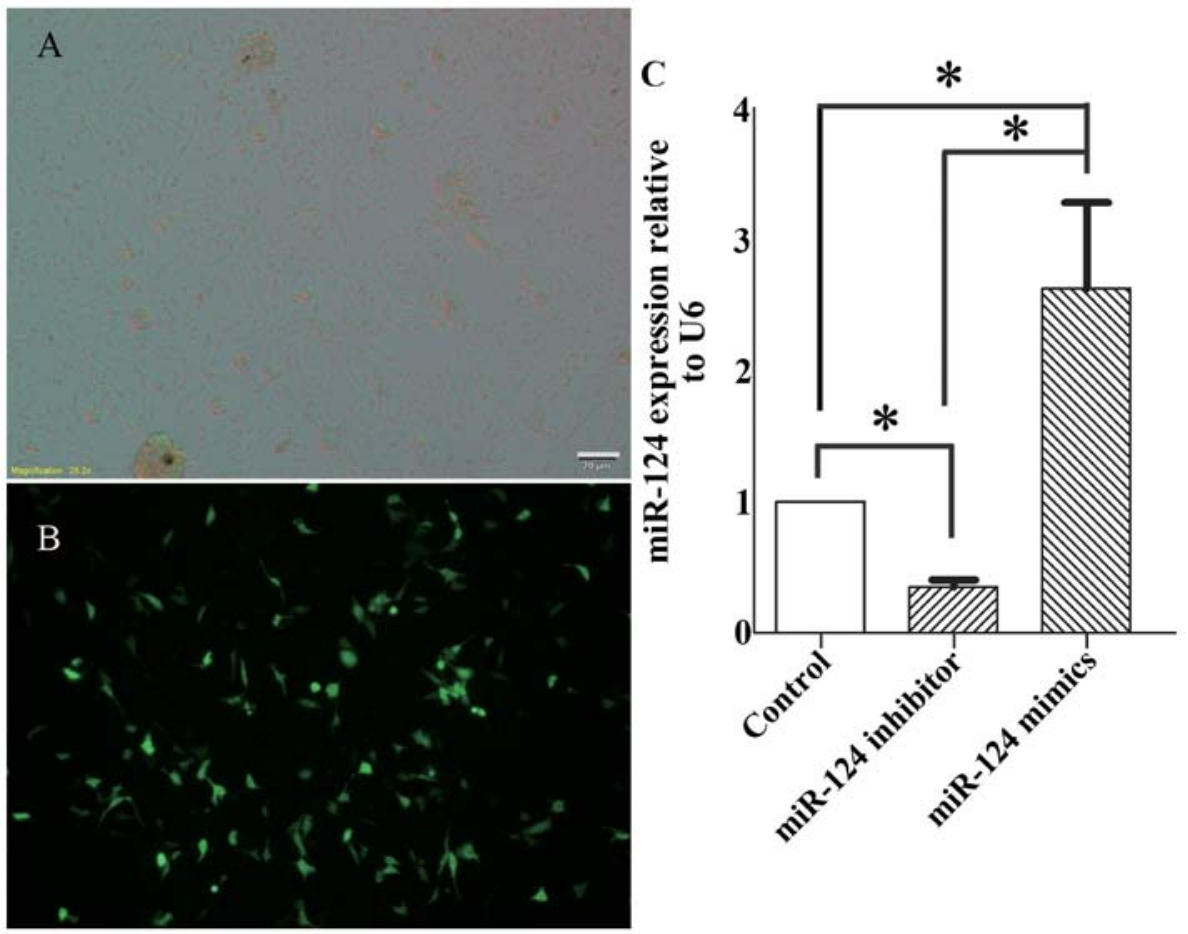

Figure 5. Transfection efficiency of inner ear neural stem cell (NSC) cultures after differentiation for 3 days. (A and B) Images of inner ear NSCs transfected with RNA oligoribonucleotides for enhanced green fluorescent protein. Scale bars, $100 \mu \mathrm{m}$. (C) miR-124 expression levels in transfected cells were determined by RT-qPCR and were normalized to U6 levels. " $\mathrm{p}<0.05$, statistically significant difference.

tion, $3.53 \pm 0.46 \%$ of the cells transfected with miR-124 mimics were Tuj1-positive cells, while the percentage of Tuj1-positive cells in the controls was $1.99 \pm 0.06 \%$ ( $<<0.05$; Fig. 6D). Moreover, the average neurite length of the Tuj1-positive cells transfected with the miR-124 mimics was $113.22 \pm 7.31 \mu \mathrm{m}$, which was significantly higher than that of the control cells
$(98.83 \pm 4.18 \mu \mathrm{m} ; \mathrm{p}<0.05)$ (Fig. 6E). By contrast, $1.05 \pm 0.25 \%$ of the cells transfected with miR-124 inhibitor were Tuj1-positive, while the percentage of Tuj1-positive cells in the controls was $1.99 \pm 0.06 \%(p<0.05$; Fig. 6D). Additionally, the average neurite length of the Tuj1-positive cells transfected with miR-124 inhibitor was reduced to $83.12 \pm 3.40 \mu \mathrm{m}$, which was 

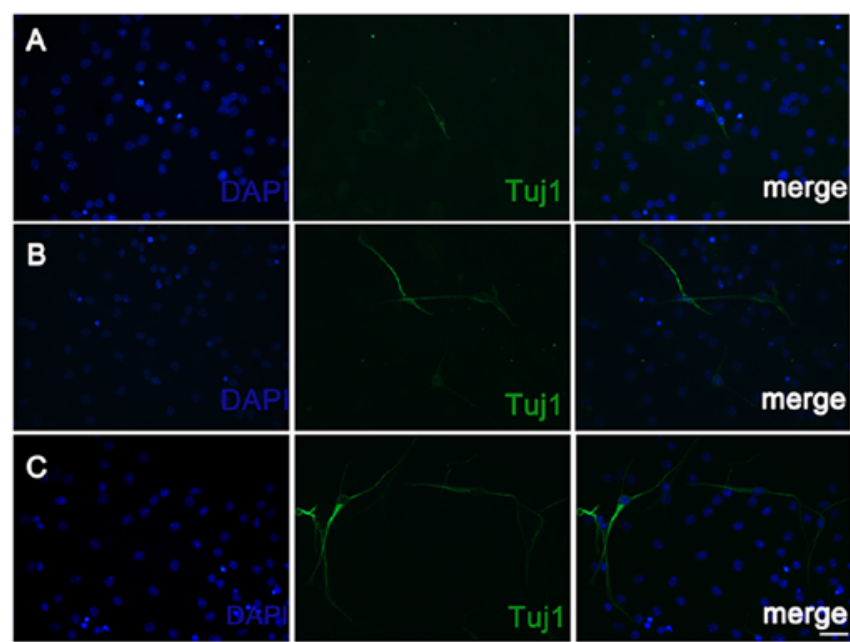

$\mathbf{E}$

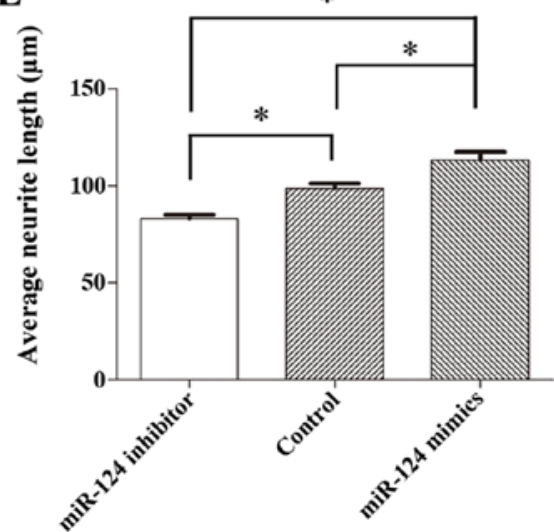

D

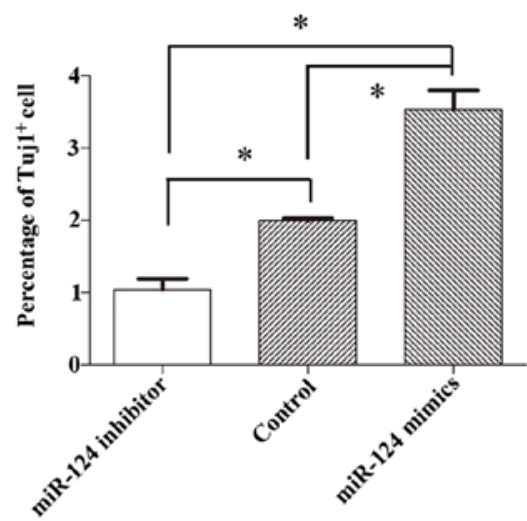

G

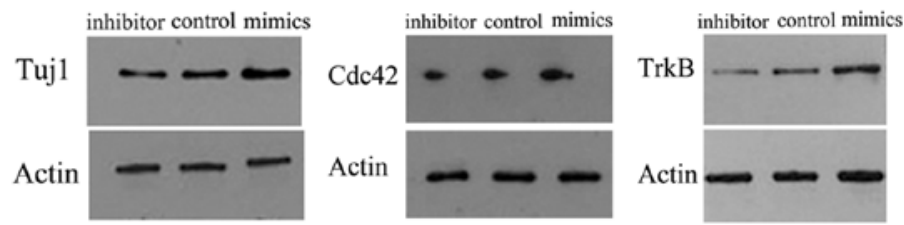

Figure 6. miR-124 promotes the neuronal differentiation of and neurite outgrowth in inner ear neural stem cells (NSCs). Inner ear NSCs transfected with (A) miR-124 inhibitor, (B) control, or (C) miR-124 mimics were differentiated into neurons for 3 days. Cells were immunostained with Tuj1 antibody (red) and nuclei were stained with 4',6-diamidino-2-phenylindole (DAPI) (blue). (D) Quantification of Tuj1-positive cells in differentiated inner ear NSCs 3 days after transfection. (E) Analysis of average neurite length examined 3 days after differentiation. (F) Western blot analysis of neuron-specific class III $\beta$-tubulin in neurons derived from inner ear NSCs 3 days after differentiation. (G) Levels of tropomyosin receptor kinase B (TrkB) and cell division control protein 42 homolog (Cdc42) in inner ear NSCs transfected with miR-124 inhibitor or miR-124 mimics. Actin was used to confirm equal loading. "p<0.05, statistically significant difference. d, days.

significantly lower than that of the controls $(98.83 \pm 4.18 \mu \mathrm{m}$; $\mathrm{p}<0.05$ ) (Fig. 6E).

We confirmed that inner ear NSCs transfected with miR-124 mimics had higher levels of neuron-specific class III $\beta$-tubulin compared to the control cells, while inner ear NSCs transfected with miR-124 inhibitor had a reduced expression of neuron-specific class III $\beta$-tubulin (Fig. 6F). These findings are in agreement with the immunofluorescence observations and confirmed the role of miR-124 in neuronal differentiation.

miR-124 affects the expression of TrkB and Cdc42 in the neuronal differentiation of mouse inner ear NSCs. TrkB and $\mathrm{Cdc} 42$ play a role in neuronal differentiation and neurite elongation. We demonstrated that $\mathrm{Cdc} 42$ and TrkB were upregulated during neuronal differentiation. Our findings revealed that miR-124 promoted the differentiation of inner ear NSCs, which led us to investigate whether miR-124 modulates TrkB and Cdc42 expression in inner ear NSCs. We evaluated the TrkB and Cdc42 expression levels in inner NSCs transfected with miR-124 by western blot analysis (Fig. 6G). Compared to the control cells, miR-124 overexpression increased Cdc42 and TrkB expression during neuronal differentiation. Conversely,
miR-124 knockdown caused a marked decrease in the TrkB and $\mathrm{Cdc} 42$ protein levels, suggesting that $\mathrm{Cdc} 42$ and TrkB act as downstream effectors of miR-124 during inner ear NSC differentiation.

\section{Discussion}

NSCs are neural progenitor cells that have the ability to self-renew, proliferate and differentiate into lineages, such as neurons, oligodendroglia and astroglia (41). Thus, NSCs, which have regenerative potential may serve as an important source for replacing damaged neurons in hearing-impaired individuals. It is therefore important to understand the differentiation pathway of inner ear NSCs into neurons. While miRNAs are generally important in neuronal development and differentiation $(19,20)$, the profiling of miRNAs in the inner ear has been a major research goal $(27,28,42,43)$. Conditional Dicer knockout mice have major defects in the inner ear, including hair cell malformations, abnormal innervations, disrupted neurosensory development and hearing loss $(29,33)$. A role of miR-96 in progressive hearing loss in mice and humans has been reported. Furthermore, miR-181a influences 
proliferation within the basilar papilla (44), while miR-200b can cause severe malformations of the auditory and vestibular labyrinth by affecting the Zeb1 pathway (45). miR-183 may affect zebrafish inner ear organization (31), as well as mouse inner ear function $(46,47)$. However, the role of miRNAs in the neuronal differentiation of mouse inner ear NSCs warrants further investigation.

In this study, we examined the expression of 6 miRNAs in the differentiation of mouse inner ear NSCs into neurons. In this study, the levels of miR-30a-5p, an established inhibitor of BDNF expression (48), increased 14 days after neuronal differentiation, suggesting that miR-30a-5p plays a role in the late stages of neuronal differentiation or neurite plasticity. In contrast to previously published results (48-53), miR-134 and miR-132 were downregulated during the neuronal differentiation of inner ear NSCs. Although miR-20a has been shown to be downregulated in the neuronal lineage differentiation of unrestricted somatic stem cells (USSCs) from cord blood and NSCs $(54,55)$, no changes in miR-20a were observed during differentiation of NSCs in our study. In addition, in our study, miR-17-5p expression increased during the first 3 days of differentiation and then increased on day 14 ; however, in a previous study, its expression decreased continued to decrease in differentiated SH-SY5Y cells (21). The differential expression of specific miRNAs may be due to specific neuronal types and time points being investigated.

In this study, we focused on the role of miR-124, which has been shown to enhance the development of mature neurons $(56,57)$ and to play a role in the neuronal differentiation of several cell lines in vivo and in vitro $(16,22,23,25,58)$. Moreover, miR-124 acts as a key mediator in regulating the differentiation of human embryonic and mesenchymal stem cells $(59,60)$. We demonstrated that miR-124 was detectable at low levels in the undifferentiated inner ear NSCs; however, its expression gradually increased and peaked on day 14 of differentiation. The overexpression of miR-124 increased the percentages of neurons and neurite length, whereas the knockdown of miR-124 had an opposite effect, demonstrating that miR-124 is important for the in vitro neuronal differentiation of inner ear NSCs.

Cdc42 is a member of the Rho GTPases and regulates both microtubules and actin filaments in a variety of cells (61-63), and plays a role in the establishment of neuronal polarity $(36,37,39,64)$. In addition, TrkB, the cognate receptor of BDNF, participates in multiple aspects of neuronal functions (65), modulating neurite outgrowth, structural plasticity and survival (36,38-40). Cdc42 functions downstream of BDNF stimulation $(36,40,66,67)$. Furthermore, BDNF activates $\mathrm{Cdc} 42$ via the Cdk5-mediated phosphorylation of TrkB, which in turn influences dendritic growth (36). TrkB and $\mathrm{Cdc} 42$ play a role in neural development and plasticity. The pregulation of TrkB and Cdc42 expression during inner ear NSC differentiation suggests that TrkB acts upstream of $\mathrm{Cdc} 42$ in the regulation of the neuronal differentiation of and neurite outgrowth in mouse inner ear NSCs.

Recently, several studies have examined the function of $\mathrm{Cdc} 42$ in dendrite and axon development, as well as its regulation by miR-124 $(58,68)$. It has been shown that miR-124 upregulates the expression of $\mathrm{Cdc} 42$ (24). Moreover, an increased TrkB level of has been shown to be accompanied by the upregulation of miR-124 in exercise-dependent neuronal differentiation in the hippocampus of adult male rats (69).

In this study, we demonstrated that miR-124 promoted neuronal differentiation and neurite outgrowth, and we investigated the effects of miR-124 on TrkB and Cdc 42 expression during the neuronal differentiation of and neurite outgrowth in inner ear NSCs. We demonstrated that TrkB and Cdc42 expression coincided with miR-124 during inner ear NSC differentiation. These results suggest that TrkB and Cdc42 may play an important role in miR-124-regulated neuronal differentiation. In support of this hypothesis, we found that miR-124 regulates their expression, raising the possibility that the effects of miR-124 on inner ear NSC differentiation and neurite outgrowth are mediated by TrkB and $\mathrm{Cdc} 42$.

Although TrkB promotes neuronal survival and plasticity (36,38-40), Cdc42 may have opposite effects on neurite outgrowth. Several studies have revealed that $\mathrm{Cdc} 42$ enhances dendritic growth or arborization $(36,37,39)$, while other studies have demonstrated negative effects $(58,68,70)$. These studies are consistent with our hypothesis, wherein $\mathrm{Cdc} 42$ promotes neurite outgrowth. In the inner ear, TrkB is enriched in cochlear neurons and plays a role in neuronal survival and structural plasticity $(38,71,72)$. In contrast to our findings and the results reported by Brors et al (73), Rac/Cdc42 inhibition has been shown to enhance neurite formation and decrease neurite length in cochlear spiral ganglion explants in neonatal rat pups (74).

In conclusion, our results suggest that miR-124 promotes the neuronal differentiation of and neurite outgrowth in inner ear NSCs by regulating TrkB and Cdc42. Future studies are required to determine the mechanisms through which miR-124 controls TrkB and $\mathrm{Cdc} 42$ expression to regulate neuronal differentiation in the mouse inner ear.

\section{Acknowledgements}

The study was supported by grants from the National Natural Science fund of China (no. 81200748).

\section{References}

1. Spoendlin H: Retrograde degeneration of the cochlear nerve. Acta Otolaryngol 79: 266-275, 1975.

2. Hawkins JE Jr: Comparative otopathology: aging, noise, and ototoxic drugs. Adv Otorhinolaryngol 20: 125-141, 1973.

3. Zhao C, Deng W and Gage FH: Mechanisms and functional implications of adult neurogenesis. Cell 132: 645-660, 2008.

4. Oshima K, Grimm CM, Corrales CE, Senn P, Martinez Monedero R, Géléoc GS, Edge A, Holt JR and Heller S: Differential distribution of stem cells in the auditory and vestibular organs of the inner ear. J Assoc Res Otolaryngol 8: 18-31, 2007.

5. Oshima K, Senn P and Heller S: Isolation of sphere-forming stem cells from the mouse inner ear. Methods Mol Biol 493: 141-162, 2009.

6. Rask-Andersen H, Boström M, Gerdin B, Kinnefors A, Nyberg G, Engstrand T, Miller JM and Lindholm D: Regeneration of human auditory nerve. In vitro/in video demonstration of neural progenitor cells in adult human and guinea pig spiral ganglion. Hear Res 203: 180-191, 2005.

7. Gu S, Jin L, Zhang F, Sarnow P and Kay MA: Biological basis for restriction of microRNA targets to the 3' untranslated region in mammalian mRNAs. Nat Struct Mol Biol 16: 144-150, 2009.

8. Lai EC: MicroRNAs are complementary to 3' UTR sequence motifs that mediate negative post-transcriptional regulation. Nat Genet 30: 363-364, 2002. 
9. Valencia-Sanchez MA, Liu J, Hannon GJ and Parker R: Control of translation and mRNA degradation by miRNAs and siRNAs. Genes Dev 20: 515-524, 2006.

10. Vaucheret H: Post-transcriptional small RNA pathways in plants: Mechanisms and regulations. Genes Dev 20: 759-771, 2006.

11. Ambros V: The functions of animal microRNAs. Nature 431: 350-355, 2004

12. Bartel DP: MicroRNAs: Genomics, biogenesis, mechanism, and function. Cell 116: 281-297, 2004.

13. Darnell DK, Kaur S, Stanislaw S, Konieczka JH, Yatskievych TA and Antin PB: MicroRNA expression during chick embryo development. Dev Dyn 235: 3156-3165, 2006.

14. Deo M, Yu JY, Chung KH, Tippens M and Turner DL: Detection of mammalian microRNA expression by in situ hybridization with RNA oligonucleotides. Dev Dyn 235: 2538-2548, 2006.

15. Kapsimali M, Kloosterman WP, de Bruijn E, Rosa F, Plasterk RH and Wilson SW: MicroRNAs show a wide diversity of expression profiles in the developing and mature central nervous system. Genome Biol 8: R173, 2007.

16. Krichevsky AM, Sonntag KC, Isacson O and Kosik KS: Specific microRNAs modulate embryonic stem cell-derived neurogenesis. Stem Cells 24: 857-864, 2006.

17. Mansfield JH, Harfe BD, Nissen R, Obenauer J, Srineel J, Chaudhuri A, Farzan-Kashani R, Zuker M, Pasquinelli AE, Ruvkun G, et al: MicroRNA-responsive 'sensor' transgenes uncover Hox-like and other developmentally regulated patterns of vertebrate microRNA expression. Nat Genet 36: 1079-1083, 2004.

18. Nelson PT, Baldwin DA, Kloosterman WP, Kauppinen S, Plasterk RH and Mourelatos Z: RAKE and LNA-ISH reveal microRNA expression and localization in archival human brain RNA 12: 187-191, 2006.

19. Fineberg SK, Kosik KS and Davidson BL: MicroRNAs potentiate neural development. Neuron 64: 303-309, 2009.

20. Gao FB: Context-dependent functions of specific microRNAs in neuronal development. Neural Dev 5: 25, 2010.

21. Beveridge NJ, Tooney PA, Carroll AP, Tran N and Cairns MJ: Down-regulation of miR-17 family expression in response to retinoic acid induced neuronal differentiation. Cell Signal 21: 1837-1845, 2009.

22. Hohjoh $\mathrm{H}$ and Fukushima T: Marked change in microRNA expression during neuronal differentiation of human teratocarcinoma NTera2D1 and mouse embryonal carcinoma P19 cells. Biochem Biophys Res Commun 362: 360-367, 2007.

23. Le MT, Xie H, Zhou B, Chia PH, Rizk P, Um M, Udolph G, Yang H, Lim B and Lodish HF: MicroRNA-125b promotes neuronal differentiation in human cells by repressing multiple targets. Mol Cell Biol 29: 5290-5305, 2009.

24. Makeyev EV, Zhang J, Carrasco MA and Maniatis T: The MicroRNA miR-124 promotes neuronal differentiation by triggering brain-specific alternative pre-mRNA splicing. Mol Cell 27: 435-448, 2007.

25. Visvanathan J, Lee S, Lee B, Lee JW and Lee SK: The microRNA miR-124 antagonizes the anti-neural REST/SCP1 pathway during embryonic CNS development. Genes Dev 21: 744-749, 2007.

26. Sacheli R, Nguyen L, Borgs L, Vandenbosch R, Bodson M, Lefebvre P and Malgrange B: Expression patterns of miR-96, miR-182 and miR-183 in the development inner ear. Gene Expr Patterns 9: 364-370, 2009.

27. Wang XR, Zhang XM, Zhen J, Zhang PX, Xu G and Jiang $H$ : MicroRNA expression in the embryonic mouse inner ear. Neuroreport 21: 611-617, 2010.

28. Weston MD, Pierce ML, Rocha-Sanchez S, Beisel KW and Soukup GA: MicroRNA gene expression in the mouse inner ear. Brain Res 1111: 95-104, 2006.

29. Friedman LM, Dror AA, Mor E, Tenne T, Toren G, Satoh T, Biesemeier DJ, Shomron N, Fekete DM, Hornstein E and Avraham KB: MicroRNAs are essential for development and function of inner ear hair cells in vertebrates. Proc Natl Acad Sci USA 106: 7915-7920, 2009.

30. Lewis MA, Quint E, Glazier AM, Fuchs H, De Angelis MH, Langford C, van Dongen S, Abreu-Goodger C, Piipari M, Redshaw N, et al: An ENU-induced mutation of miR-96 associated with progressive hearing loss in mice. Nat Genet 41: 614-618, 2009

31. Li H, Kloosterman W and Fekete DM: MicroRNA-183 family members regulate sensorineural fates in the inner ear. J Neurosci 30: 3254-3263, 2010.
32. Mencía A, Modamio-Høybjør S, Redshaw N, Morín M, Mayo-Merino F, Olavarrieta L, Aguirre LA, del Castillo I, Steel KP, Dalmay T, et al: Mutations in the seed region of human miR-96 are responsible for nonsyndromic progressive hearing loss. Nat Genet 41: 609-613, 2009.

33. Soukup GA, Fritzsch B, Pierce ML, Weston MD, Jahan I, McManus MT and Harfe BD: Residual microRNA expression dictates the extent of inner ear development in conditional Dicer knockout mice. Dev Biol 328: 328-341, 2009.

34. Martinez-Monedero R, Yi E, Oshima K, Glowatzki E and Edge AS: Differentiation of inner ear stem cells to functional sensory neurons. Dev Neurobiol 68: 669-684, 2008.

35. Roehm PC, Xu N, Woodson EA, Green SH and Hansen MR: Membrane depolarization inhibits spiral ganglion neurite growth via activation of multiple types of voltage sensitive calcium channels and calpain. Mol Cell Neurosci 37: 376-387, 2008.

36. Cheung ZH, Chin WH, Chen Y, Ng YP and Ip NY: Cdk5 is involved in BDNF-stimulated dendritic growth in hippocampal neurons. PLoS Biol 5: e63, 2007.

37. Endo M, Antonyak MA and Cerione RA: Cdc42-mTOR signaling pathway controls Hes5 and Pax6 expression in retinoic aciddependent neural differentiation. J Biol Chem 284: 5107-5118, 2009.

38. Sciarretta C, Fritzsch B, Beisel K, Rocha-Sanchez SM,Buniello A, Horn JM and Minichiello L: PLC $\gamma$-activated signalling is essential for TrkB mediated sensory neuron structural plasticity. BMC Dev Biol 10: 103, 2010.

39. Sosa L, Dupraz S, Laurino L, Bollati F, Bisbal M, Cáceres A, Pfenninger KH and Quiroga S: IGF-1 receptor is essential for the establishment of hippocampal neuronal polarity. Nat Neurosci 9: 993-995, 2006.

40. Lai KO, Wong AS, Cheung MC, Xu P, Liang Z, Lok KC, Xie H, Palko ME, Yung WH, Tessarollo L, et al: TrkB phosphorylation by Cdk5 is required for activity-dependent structural plasticity and spatial memory. Nat Neurosci 15: 1506-1515, 2012.

41. Gage FH: Mammalian neural stem cells. Science 287: 1433-1438, 2000.

42. Elkan-Miller T, Ulitsky I, Hertzano R, Rudnicki A, Dror AA, Lenz DR, Elkon R, Irmler M, Beckers J, Shamir R and Avraham KB: Integration of transcriptomics, proteomics, and microRNA analyses reveals novel microRNA regulation of targets in the mammalian inner ear. PLoS One 6: e18195, 2011.

43. Patel M, Cai Q, Ding D, Salvi R, Hu Z and Hu BH: The miR-183/ Taok1 target pair is implicated in cochlear responses to acoustic trauma. PLoS One 8: e58471, 2013.

44. Frucht CS, Santos-Sacchi J and Navaratnam DS: MicroRNA181a plays a key role in hair cell regeneration in the avian auditory epithelium. Neurosci Lett 493: 44-48, 2011.

45. Hertzano R, Elkon R, Kurima K, Morrisson A, Chan SL, Sallin M, Biedlingmaier A, Darling DS, Griffith AJ, Eisenman DJ and Strome SE: Cell type-specific transcriptome analysis reveals a major role for Zeb1 and miR-200b in mouse inner ear morphogenesis. PLoS Genet 7: e1002309, 2011.

46. Gu C, Li X, Tan Q, Wang Z, Chen L and Liu Y: MiR-183 family regulates chloride intracellular channel 5 expression in inner ear hair cells. Toxicol In Vitro 27: 486-491, 2013.

47. Wang XR, Zhang XM, Du J and Jiang H: MicroRNA-182 regulates otocyst-derived cell differentiation and targets T-box1 gene. Hear Res 286: 55-63, 2012.

48. Mellios N, Huang HS, Grigorenko A, Rogaev E and Akbarian S: A set of differentially expressed miRNAs, including miR-30a-5p, act as post-transcriptional inhibitors of BDNF in prefrontal cortex. Hum Mol Genet 17: 3030-3042, 2008

49. Gaughwin P, Ciesla M, Yang H, Lim B and Brundin P: Stage-specific modulation of cortical neuronal development by Mmu-miR-134. Cereb Cortex 21: 1857-1869, 2011.

50. Mellios N, Sugihara H, Castro J, Banerjee A, Le C, Kumar A Crawford B, Strathmann J, Tropea D, Levine SS, et al: miR-132, an experience-dependent microRNA, is essential for visual cortex plasticity. Nat Neurosci 14: 1240-1242, 2011.

51. Clovis YM, Enard W, Marinaro F, Huttner WB and De Pietri Tonelli D: Convergent repression of Foxp2 3'UTR by miR-9 and miR-132 in embryonic mouse neocortex: Implications for radial migration of neurons. Development 139: 3332-3342, 2012.

52. Lin LF, Chiu SP, Wu MJ, Chen PY and Yen JH: Luteolin induces microRNA-132 expression and modulates neurite outgrowth in PC12 cells. PLoS One 7: e43304, 2012. 
53. Pathania M, Torres-Reveron J, Yan L, Kimura T, Lin TV, Gordon V, Teng ZQ, Zhao X, Fulga TA, Van Vactor D and Bordey A: miR-132 enhances dendritic morphogenesis, spine density, synaptic integration, and survival of newborn olfactory bulb neurons. PLoS One 7: e38174, 2012.

54. Aranha MM, Santos DM, Xavier JM, Low WC, Steer CJ, Solá S and Rodrigues CM: Apoptosis-associated microRNAs are modulated in mouse, rat and human neural differentiation. BMC Genomics 11: 514, 2010.

55. Trompeter HI, Abbad H, Iwaniuk KM, Hafner M, Renwick N, Tuschl T, Schira J, Müller HW and Wernet P: MicroRNAs MiR-17, MiR-20a, and MiR-106b act in concert to modulate E2F activity on cell cycle arrest during neuronal lineage differentiation of USSC. PLoS One 6: e16138, 2011.

56. Miska EA, Alvarez-Saavedra E, Townsend M, Yoshii A, Sestan N, Rakic P, Constantine-Paton M and Horvitz HR: Microarray analysis of microRNA expression in the developing mammalian brain. Genome Biol 5: R68, 2004.

57. Smirnova L, Gräfe A, Seiler A, Schumacher S, Nitsch R and Wulczyn FG: Regulation of miRNA expression during neural cell specification. Eur J Neurosci 21: 1469-1477, 2005.

58. Yu JY, Chung KH, Deo M, Thompson RC and Turner DL: MicroRNA miR-124 regulates neurite outgrowth during neuronal differentiation. Exp Cell Res 314: 2618-2633, 2008.

59. Lee MR, Kim JS and Kim KS: miR-124a is important for migratory cell fate transition during gastrulation of human embryonic stem cells. Stem Cells 28: 1550-1559, 2010.

60. Maisel M, Habisch HJ, Royer L, Herr A, Milosevic J, Hermann A, Liebau S, Brenner R, Schwarz J, Schroeder M and Storch A: Genome-wide expression profiling and functional network analysis upon neuroectodermal conversion of human mesenchymal stem cells suggest HIF-1 and miR-124a as important regulators. Exp Cell Res 316: 2760-2778, 2010.

61. Hall A: Rho GTPases and the actin cytoskeleton. Science 279: 509-514, 1998

62. Jaffe AB and Hall A: Rho GTPases: Biochemistry and biology. Annu Rev Cell Dev Biol 21: 247-269, 2005.

63. Li E, Stupack D, Bokoch GM and Nemerow GR: Adenovirus endocytosis requires actin cytoskeleton reorganization mediated by Rho family GTPases. J Virol 72: 8806-8812, 1998.

64. Schwamborn JC and Püschel AW: The sequential activity of the GTPases Rap1B and Cdc42 determines neuronal polarity. Nat Neurosci 7: 923-929, 2004
65. Huang EJ and Reichardt LF: Trk receptors: Roles in neuronal signal transduction. Annu Rev Biochem 72: 609-642, 2003.

66. Chen TJ, Gehler S, Shaw AE, Bamburg JR and Letourneau PC: $\mathrm{Cdc} 42$ participates in the regulation of $\mathrm{ADF} /$ cofilin and retinal growth cone filopodia by brain derived neurotrophic factor. J Neurobiol 66: 103-114, 2006.

67. Shen W, Wu B, Zhang Z, Dou Y, Rao ZR, Chen YR and Duan S: Activity-induced rapid synaptic maturation mediated by presynaptic cdc42 signaling. Neuron 50: 401-414, 2006.

68. Franke K, Otto W, Johannes S, Baumgart J, Nitsch R and Schumacher S: miR-124-regulated RhoG reduces neuronal process complexity via ELMO/Dock180/Rac1 and Cdc42 signalling. EMBO J 31: 2908-2921, 2012.

69. Mojtahedi S, Kordi MR, Hosseini SE, Omran SF and Soleimani M: Effect of treadmill running on the expression of genes that are involved in neuronal differentiation in the hippocampus of adult male rats. Cell Biol Int 37: 276-283, 2013.

70. Hing H, Xiao J, Harden N, Lim L and Zipursky SL: Pak functions downstream of Dock to regulate photoreceptor axon guidance in Drosophila. Cell 97: 853-863, 1999.

71. Schimmang T, Minichiello L, Vazquez E, San Jose I, Giraldez F, Klein R and Represa J: Developing inner ear sensory neurons require TrkB and TrkC receptors for innervation of their peripheral targets. Development 121: 3381-3391, 1995.

72. Schimmang T, Tan J, Müller M, Zimmermann U, Rohbock K, Kôpschall I, Limberger A, Minichiello L and Knipper M: Lack of Bdnf and TrkB signalling in the postnatal cochlea leads to a spatial reshaping of innervation along the tonotopic axis and hearing loss. Development 130: 4741-4750, 2003.

73. Brors D, Aletsee C, Dazert S, Huverstuhl J, Ryan AF and Bodmer D: Clostridium difficile toxin B, an inhibitor of the small GTPases Rho, Rac and Cdc42, influences spiral ganglion neurite outgrowth. Acta Otolaryngol 123: 20-25, 2003

74. Mullen LM, Pak KK, Chavez E, Kondo K, Brand Y and Ryan AF: Ras/p38 and PI3K/Akt but not Mek/Erk signaling mediate BDNF-induced neurite formation on neonatal cochlear spiral ganglion explants. Brain Res 1430: 25-34, 2012. 